\title{
AS MUDANÇAS FUNCIONAIS NO PROCESSO DE PROTETIZAÇÃO PARA AMPUTAÇÃO DE PUNHO COM PRÓTESE PERSONALIZADA EM IMPRESSORA 3D
}

\section{Raissa Rodrigues Pereira Lima}

Acadêmica do Centro Universitário do Estado do Pará - CESUPA, Brasil.

\section{Gabriela Trindade Felix}

Acadêmica do Centro Universitário do Estado do ParáCESUPA, Brasil

\section{Wellington Pinheiro de Oliveira} Molecular pela Universidade Federal do Pará (UFPA). Docente do curso de Fisioterapia e Medicina do Centro Universitário do Pará - CESUPA, Brasil.
Fisioterapeuta, mestre em Genética e Biologia

RESUMO: Avaliar as mudanças funcionais do membro superior em paciente amputado de punho utilizando prótese personalizada em impressora 3D. Trata-se de um estudo de caso referente ao atendimento fisioterapêutico de um paciente com amputação de punho do tipo infecciosa e avaliação da função de apertar, segurar, pinça e movimento grosseiro do membro superior afetado antes e após um tratamento protético de dez sessões. Após o treinamento protético foi feita a reavaliação, sendo possível observar que a funcionalidade do membro superior do participante melhorou com o uso da prótese nos seguintes testes: apertar ( 2 pontos); segurar (1,5 ponto); e movimento grosseiro (3 pontos). No entanto, pelo fato de a prótese fazer apenas o movimento de preensão grossa, não foi possível ganhar funcionalidade no teste de pinça (0 ponto). As mudanças funcionais avaliadas demonstraram melhora significativa na funcionalidade, referente aos movimentos de apertar, segurar e movimento grosseiro.

PALAVRAS-CHAVE: Amputação; Membros Artificiais; Desempenho Físico Funcional; Fisioterapia.

\section{FUNCTIONAL CHANGES IN THE PROSTHESIS PROCESS FOR HAND AMPUTATION BY 3D PERSONALIZED PROSTHESIS PRINTER}

\begin{abstract}
Functional changes of a higher body member in patients with hand amputation are evaluated, coupled to the use of personalized prosthesis by 3D printer. The case study refers to physiotherapeutic care of a patient with hand amputation due to infection and the evaluation of the holding, tightening, clamp and coarse movement of the higher member prior to and after a 10 -session prosthetic treatment. Revaluation after prosthetic training revealed that the functionality of the participant's upper member improved with the use of the prosthesis when assessed by the following tests: holding ( 2 points), tightening (1.5 points); gross movement (3 points). However, since prosthesis only undertook coarse holding, there was no improvement in the tweeze test ( 0 points). Functional changes showed significant improvement in functionality with regard to movements of holding, tightening and coarse movement.
\end{abstract}

KEYWORDS: Amputation; Artificial limbs; Physical functional performance; Physical Therapy Specialty.

\section{INTRODUÇÃO}

Amputação é o processo de separação de parte do organismo,

Autor correspondente

Raissa Rodrigues Pereira Lima

raissarplima@hotmail.com podendo esta ser um membro ou outra parte do corpo. No Brasil, estimase que a incidência de amputações seja de 13,9 por 100.000 habitantes/ 
ano e, dentre as indicações para o procedimento de amputação, estão traumas, tumores malignos, infecções, problemas vasculares e deformidades congênitas ${ }^{1}$.

O paciente amputado pode apresentar diversas complicações físicas e modificações bruscas em sua vida que alteram seu comportamento, hábitos e atividades, podendo impossibilitar o progresso de cada uma das fases da reabilitação. Além disso, isso pode causar no indivíduo uma súbita, e, na maioria das vezes, inesperada, alteração da imagem corporal, sendo necessária uma reintegração desta imagem ao seu novo esquema corporal ${ }^{2,3}$.

Visando à reintegração corporal mais eficaz e produtiva, o paciente deve primeiramente aceitar sua perda física. A aceitação da condição atual é essencial para que o paciente possa integrar as funções de uma prótese aos movimentos e à funcionalidade do membro amputado, permitindo o domínio e o controle de seus movimentos, identificando suas limitações e convivendo com elas, desenvolvendo estratégias no seu dia a dia que lhe possibilitem maior autonomia ${ }^{4}$.

Independente da causa da amputação, o fisioterapeuta tem função importante no programa de reabilitação, devendo seguir objetivos precisos e viáveis. Sendo assim, é importante que haja uma avaliação minuciosa da funcionalidade do paciente para que se definam as capacidades residuais e potenciais para se estabelecer meios, metas e parâmetros adequados para esse processo, ${ }^{5,6}$.

A prótese surge como um dispositivo destinado a substituir um órgão ou um membro que foi gravemente acometido. Como tais instrumentos não são naturais do corpo, há a necessidade de um processo de adaptação com o objetivo de solucionar a deficiência presente. Essa experiência pode ocasionar no indivíduo uma série de sensações, fazendo-se necessário o constante apoio da equipe de saúde ${ }^{7,8}$.

A tecnologia de prototipagem rápida (impressão 3D) vem crescendo na produção de próteses mecânicas as quais são compostas de ácido polilático (PLA), um polímero economicamente viável para produções em pequena e larga escala, tornando a prótese de baixo custo. O material sofre termoformação, quando exposto a temperaturas acima de $60^{\circ} \mathrm{C}$. Para se imprimir uma prótese $3 \mathrm{D}$ que seja funcional, são necessárias as medidas do membro, sendo possível criar ou editar modelos já existentes e esse processo permite a adaptação adequada ao corpo $^{9,10}$.

Nesse contexto, a atuação do fisioterapeuta promove a reabilitação e adaptação adequada à prótese para que haja segurança, controle e estabilidade durante a utilização desta, a fim de que recupere a funcionalidade do membro amputado, trazendo maior independência ao paciente $^{11}$.

O programa deve incluir treinamento préprotético e protético. No período pré-protético, o cuidado é focado para manter o movimento normal, força do membro amputado, moldar o coto para o encaixe da prótese, tratar possíveis sintomas de membro fantasma e cuidar de edemas e cicatrizes na região. Após isso, o treinamento protético visa habilitar o paciente para o controle da prótese, com treinos repetitivos de atividades de vida diárias ${ }^{12}$.

Sendo assim, o objetivo desta pesquisa foi avaliar as mudanças funcionais do membro superior em paciente amputado de punho utilizando prótese personalizada em impressora 3D.

\section{DESCRIÇÃO DO CASO}

Trata-se de um estudo de caso referente ao atendimento fisioterapêutico de paciente com desarticulação de punho do tipo infecciosa, submetido a tratamento protético de dez sessões na Clínica Escola de Fisioterapia do CESUPA.

$O$ participante foi submetido à avaliação fisioterapêutica específica para paciente amputado, e foram coletados dados de identificação, anamnese, exame físico geral e exame específico do coto que avaliou a cicatrização, presença de deformidades, membro fantasma, dor fantasma, avaliação vascular no local, palpação, amplitude de movimento e foram feitas as medidas necessárias para a confecção da prótese ${ }^{13}$.

O voluntário, de 48 anos, sexo masculino, divorciado, natural de Belém e com ensino médio completo, relatou que sofrera agressão por arma branca no punho esquerdo em 2003, que, por falta de cuidados, evoluiu para infecção grave e posterior amputação. No exame físico geral, apresentou dificuldade em realizar 
tarefas que exigiam a utilização das duas mãos. No exame do coto (Figura 1), não apresentou alterações de pele ou deformidades, apresentando apenas aderência na cicatriz, coceira em membro fantasma e dor ao bater o coto. Além disso, não tinha alterações de tônus, ou de força no membro superior esquerdo, manifestando apenas leve hipotrofia em antebraço. Foi mensurado o comprimento do membro superior esquerdo $(54 \mathrm{~cm})$, braço $(28 \mathrm{~cm})$, antebraço $(24 \mathrm{~cm})$ e da extremidade do coto $(6,5 \mathrm{~cm})$ e realizada a perimetria das regiões média $(28 \mathrm{~cm})$ e distal do braço $(27 \mathrm{~cm})$, linha articular do cotovelo $(25 \mathrm{~cm})$, das regióes proximal $(22 \mathrm{~cm})$, média $(18 \mathrm{~cm})$ e distal $(17 \mathrm{~cm})$ do antebraço. Por último, o participante listou as atividades que almejava conseguir fazer com a prótese ${ }^{14}$.
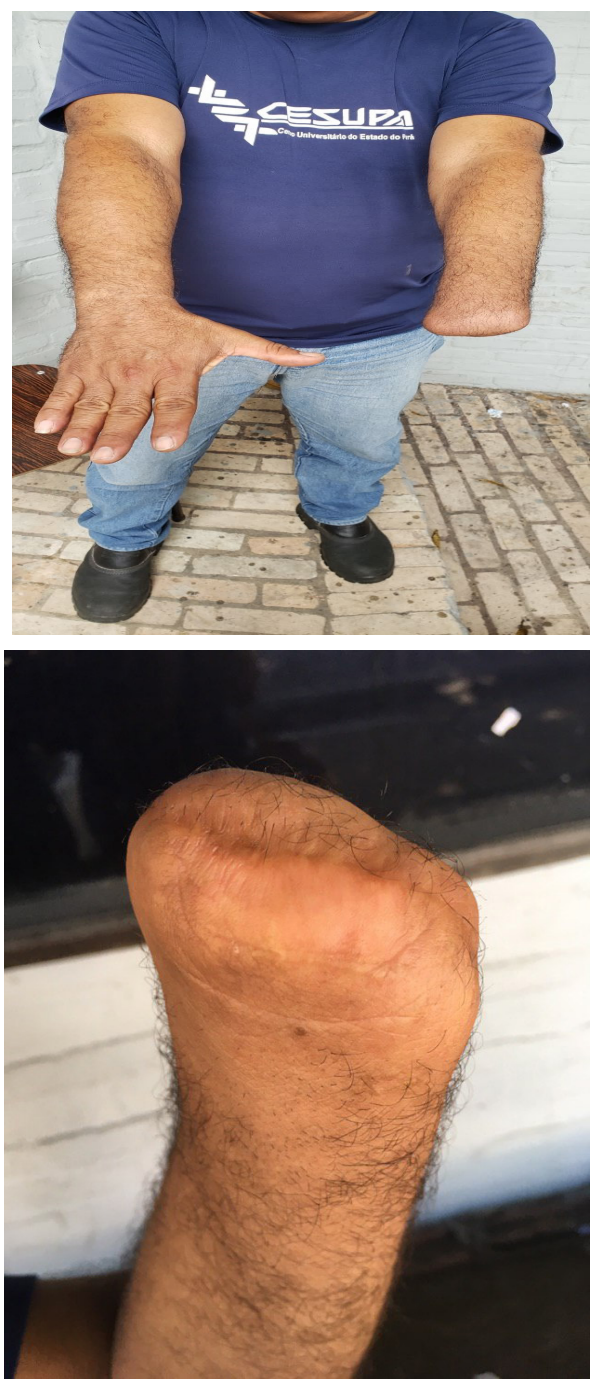

Figura 1. Exame do coto Fonte: Protocolo de pesquisa (2018).

A partir da perimetria, a prótese foi confeccionada no programa Fusion 360 e impressa na impressora 3D BuilderBox em PLA, material que foi escolhido por sua capacidade de deformação e adaptação no paciente. A prótese foi impressa em partes separadas, portanto, foi necessário fazer a montagem e modelagem da mesma.

O mecanismo da prótese ocorre inicialmente pelo fato de todas as falanges serem articuladas, permitindo o movimento de preensão. Para se promover tal movimento, são presos fios de nylon em cada falange distal e que passam por dentro da prótese para serem fixados na região do braço. Quando o indivíduo realiza a flexão de cotovelo, os fios são tensionados de maneira que puxem as falanges, promovendo, assim, a flexão dos dedos. Já quando se realiza a extensão de cotovelo, a tensão nos fios diminui e os elásticos que são colocados em cada articulação, correspondente às interfalangeanas, realizam a extensão dos dedos (Figura 2).

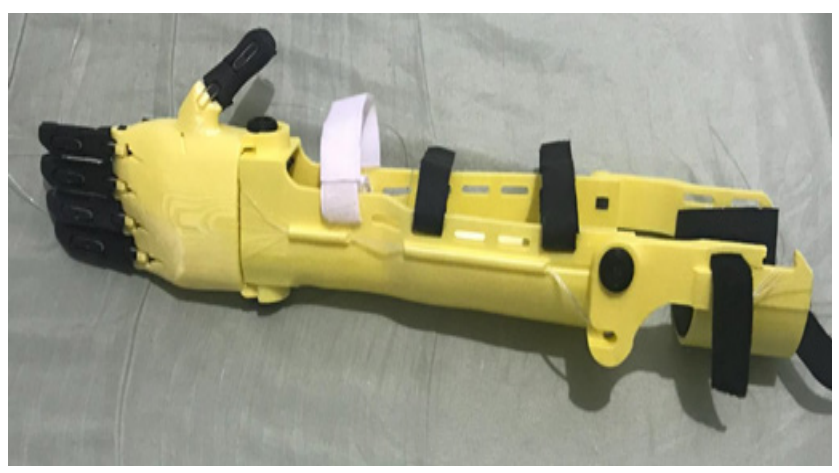

Figura 2. Prótese pronta e moldada. Fonte: Protocolo de pesquisa (2018).

Foi avaliada também a função motora por meio do instrumento, validado apenas em inglês, denominado Action Research Arm Test (Teste de Pesquisa - Ação do Braço, em português), por meio das seguintes ações: apertar, segurar, pinça e movimento grosseiro; é dado um score de 0 a 3 para cada função, sendo $0=$ sem movimento, $1=$ movimento de performance parcial, $2=$ movimento completo mas com anormalidades e $3=$ movimento com performance normal ${ }^{15}$.

Uma vez confeccionada a prótese, foi aplicado o protocolo de treinamento protético sugerido por Brasil (2013), de forma adaptada, dividido em quatro fases (Figura 3). A primeira fase se constituiu de uma sessão, que consistiu na adaptação e encaixe da prótese no paciente, no entanto foram verificados um encaixe inadequado para o cotovelo e antebraço bem como uma fragilidade na espessura das partes planas, levando à necessidade de reimpressão da prótese e repetição da primeira fase ${ }^{16}$. 


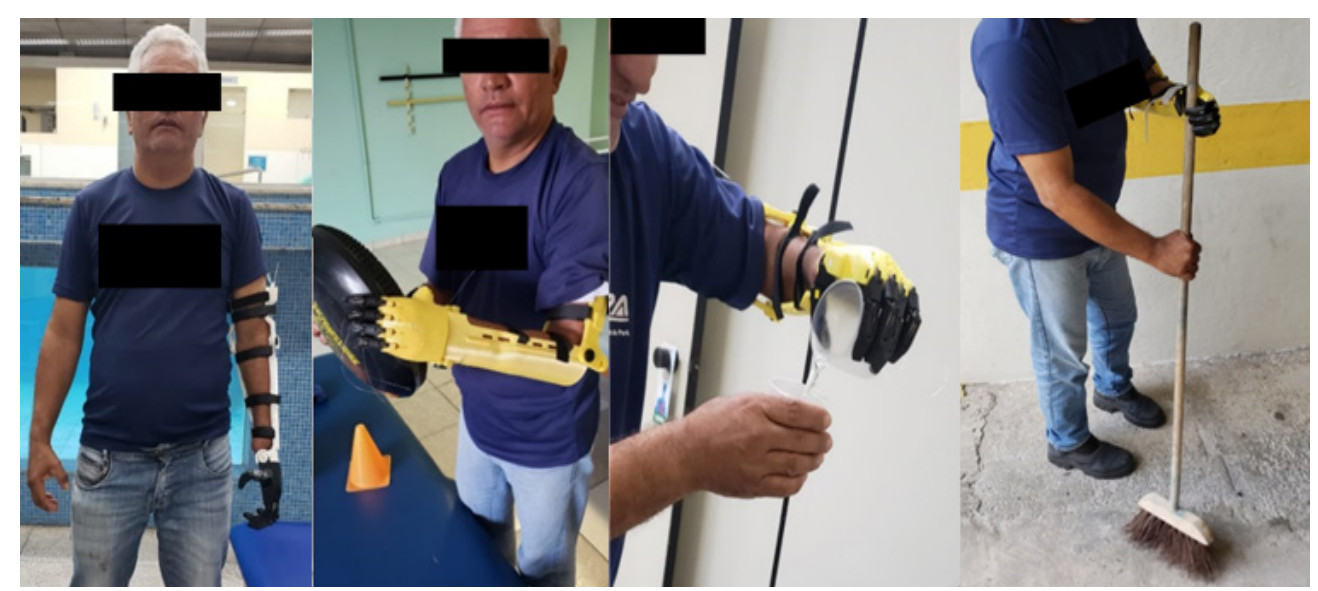

Figura 4. Fases do treinamento protético

Fonte: Protocolo de pesquisa (2019).

$\mathrm{Na}$ segunda fase, constituída de três sessões, houve adaptação do paciente aos movimentos necessários para o funcionamento da prótese, como flexão e extensão de cotovelo para realizar flexão e extensão de dedos. Já a terceira fase, realizada em três sessões, teve como foco o treinamento com objetos de pesos e tamanhos diferentes como bloco de madeira, garrafa de álcool, copo, travesseiro, disco de propriocepção, cadeira, vassoura, halter e caneleira. Por fim, a quarta fase, conduzida em três sessões, objetivou adaptar o paciente às atividades de vida diária, levando-se em consideração a sua lista apresentada na Tabela 2 .

Além disso, foi feita a reavaliação para se coletar os dados referentes ao resultado do treinamento protético.

O Quadro 1 apresenta os dados da avaliação por meio do Action Research Arm Test antes e depois do treinamento protético, que demonstrou que, sem a prótese, o paciente não conseguia realizar nenhum dos domínios do teste, resultando, assim, na baixa funcionalidade do membro.

Quadro 1. Action Research Arm Test antes e depois do treinamento protético

(Continua)

\begin{tabular}{|c|c|c|}
\hline \multicolumn{3}{|l|}{ Nome do paciente: MFSN } \\
\hline \multicolumn{3}{|c|}{ Pontuação de atividade } \\
\hline Apertar & Antes & Depois \\
\hline $\begin{array}{l}\text { 1. Bloco, madeira, } 10 \mathrm{~cm} \text { cubo } \\
\text { Pegar um bloco de } 10 \mathrm{~cm}\end{array}$ & 0 & 2 \\
\hline $\begin{array}{l}\text { 2. Bloco, madeira, } 2.5 \mathrm{~cm} \text { cubo } \\
\text { Pegar um bloco de } 2.5 \mathrm{~cm}\end{array}$ & 0 & 2 \\
\hline 3. Bloco, madeira, $5 \mathrm{~cm}$ cubo & 0 & 2 \\
\hline 5. Bola, $7.5 \mathrm{~cm}$ diâmetro & 0 & 2 \\
\hline 6. Pedra $10 \times 2.5 \times 1 \mathrm{~cm}$ & 0 & 2 \\
\hline Segurar & Antes & Depois \\
\hline 1. Derramar água de copo para copo & 0 & 2 \\
\hline 2. Tubo $2.25 \mathrm{~cm}$ & 0 & 2 \\
\hline 3. Tubo $1 \times 16 \mathrm{~cm}$ & 0 & 2 \\
\hline 4. Rosca sobre parafuso ( $3.5 \mathrm{~cm}$ diâmetro) & 0 & 0 \\
\hline Pinça & & \\
\hline 1. Rolamento de esfera, $6 \mathrm{~mm}, 3^{\circ}$ dedo e polegar & 0 & 0 \\
\hline
\end{tabular}


(Conclusão)

\begin{tabular}{|c|c|c|}
\hline \multicolumn{3}{|l|}{ Nome do paciente: MFSN } \\
\hline \multicolumn{3}{|c|}{ Pontuação de atividade } \\
\hline 2. Mármore, $1.5 \mathrm{~cm}$, dedo indicador e polegar & 0 & 0 \\
\hline 3. Rolamento de esfera $2^{\circ}$ dedo e polegar & 0 & 0 \\
\hline 4. Rolamento de esfera $1^{\circ}$ dedo e polegar & 0 & 0 \\
\hline 5. Mármore $3^{\circ}$ dedo e polegar & 0 & 0 \\
\hline 6. Mármore $2^{\circ}$ dedo e polegar & 0 & 0 \\
\hline Movimento grosseiro & Antes & Depois \\
\hline 1. Colocar a mão atrás da cabeça & 0 & 3 \\
\hline 2. Se pontuação $=0$, total $=0$ e termina & 0 & 3 \\
\hline 3. Colocar a mão no topo da cabeça & 0 & 3 \\
\hline 4. Mão até a boca & 0 & 3 \\
\hline
\end{tabular}

Fonte: Protocolo de pesquisa (2019).

Após o treinamento, foi possível observar que a funcionalidade do membro superior do participante melhorou nos seguintes testes: apertar de 0 (antes) para 2 pontos (depois); segurar de 0 para 1,5 ponto; e movimento grosseiro de 0 para 3 pontos. No entanto, pelo fato de a prótese fazer apenas o movimento de preensão grossa, não foi possível ganhar funcionalidade no teste de pinça.

O Quadro 2 apresenta a lista, feita pelo paciente, de expectativas quanto ao uso da prótese, com tarefas que normalmente executava com dificuldade ou que não executava e esperava que isso melhorasse após o tratamento. Também expõe a relação de expectativas alcançadas, e é possível observar um êxito em $50 \%$ das atividades almejadas pelo paciente, como, por exemplo, "usar vassoura", "dirigir ou andar de bicicleta", "segurar a enxada para capinar ou bater massa", "em um jogo de futebol, bater um lateral", "Andar de mãos dadas usando o lado esquerdo". No entanto, algumas ações da lista não puderam ser avaliadas pela falta dos recursos necessários ou possíveis no ambiente da clínica escola, como, por exemplo, "sentar um tijolo", "segurar no guidão de moto", "descamar peixe", "tratar de frango", "tirar bife".

Apesar de a prótese mecânica trazer diversos benefícios ao paciente, foi observado que há ainda algumas limitações em relação à utilização da mesma, como a restrição de supinação e pronação de antebraço, pois a região do cotovelo é fixa, permitindo apenas os movimentos de flexão e extensão; todos os movimentos de punho, mantendo sempre a mão na posição neutra; (parece deslocado aqui) limitação para força de preensão, conseguindo carregar apenas objetos mais leves, em razão de os fios de nylon não gerarem tensão suficiente para promoverem a força de preensão necessária; limitação quanto à amplitude de movimento, tendo que, em algumas situações, adotar posturas não funcionais de tronco e membros inferiores para conseguir o posicionamento adequado para a prótese pegar o objeto. Além disso, observaram-se limitações relacionadas à execução de movimentos finos, limitando a realização de algumas tarefas básicas do dia a dia.

Após todas as fases do treinamento e da adaptação, foi confeccionada uma prótese definitiva para que o paciente pudesse utilizá-la no seu dia a dia, de acordo com sua necessidade.

O estudo de caso foi idealizado e realizado de acordo com as normas de pesquisa envolvendo seres humanos descritas na resolução 466/12 do Conselho Nacional de Saúde (CNS), após o aceite do orientador e do Centro Universitário do Estado do Pará (CESUPA) onde foi realizada a pesquisa e aprovação pelo Comitê de Ética e Pesquisa do CESUPA (parecer n ${ }^{0} 3.353 .713$ ). O paciente foi informado sobre o objetivo e procedimentos da pesquisa, por meio da leitura do Termo de Consentimento Livre e Esclarecido - TCLE, momento que foram esclarecidas todas as dúvidas do paciente sobre o estudo a ser realizado e, em seguida foi assinado, conforme aceitação deste. 
Quadro 2. Lista de expectativas almejadas e alcançadas

\begin{tabular}{|cl|c|}
\hline \multicolumn{2}{|c|}{ ALMEJADAS } & ALCANÇADAS \\
\hline 1. & Usar vassoura & $\mathbf{X}$ \\
\hline 2. & Sentar um tijolo & Não avaliado \\
\hline 3. & Segurar no guidão de moto & Não avaliado \\
\hline 4. & Dirigir ou andar de bicicleta & $\mathbf{X}$ \\
\hline & \multicolumn{1}{|c|}{ ALMEJADAS } & ALCANÇADAS \\
\hline & Nescamar peixe & Não avaliado \\
\hline 5. & Tratar de frango & Não avaliado \\
\hline 6. & Tirar bife & Não avaliado \\
\hline 7. & $\begin{array}{l}\text { Segurar a enxada para capinar ou } \\
\text { bater massa }\end{array}$ \\
\hline 8. & $\begin{array}{l}\text { Em um jogo de futebol, bater um } \\
\text { lateral }\end{array}$ & $\mathbf{X}$ \\
\hline 9. & $\begin{array}{l}\text { Andar de mãos dadas usando o } \\
\text { lado esquerdo }\end{array}$ & $\mathbf{X}$ \\
\hline
\end{tabular}

Fonte: Protocolo de pesquisa (2019).

\section{DISCUSSÃO}

Independente da causa ou tipo da amputação, o fisioterapeuta é de extrema importância para a reabilitação do paciente, assim como o início precoce do tratamento influencia o resultado final da reabilitação ${ }^{16}$.

Para contribuir com a reabilitação do paciente amputado, a protetização tornou-se uma alternativa. Para isso, a utilização de impressoras 3D, nos últimos anos, vem se mostrando uma nova forma de se pensar a produção de próteses ${ }^{17}$.

A presente pesquisa demonstrou a vantagem da impressão 3D, uma vez que os protótipos são produzidos com maior agilidade, em alta diversidade de materiais, além de serem extremamente customizáveis, haja vista que utilizam as medidas e condições do paciente, o que pode levar à melhor adaptação da prótese e, assim, ao maior bem-estar, qualidade de vida e reabilitação do paciente.

De acordo com o estudo de Magnusson et al, os usuários de prótese apresentam normalmente uma qualidade de vida mais baixa quanto à saúde física, psicológica e de domínios ambientais, quando comparado com indivíduos que não possuem incapacidade, corroborando, portanto, a importância da lista de expectativas solicitada ao paciente no início do tratamento, que permitiu adaptar o trabalho para as reais necessidades do mesmo, impactando positivamente na sua qualidade de vida ${ }^{18}$.
No estudo, destacou-se também a melhora da funcionalidade do paciente após a adaptação da prótese mecânica, a qual promoveu a capacidade de apertar e segurar objetos, trazendo a possibilidade de executar atividades que não realizava antes, como observado também no estudo de Guisheng Xu et al., que avaliaram a funcionalidade de uma criança após a utilização da prótese, e foi observada melhora satisfatória em várias atividades de vida diária como comer, escrever, vestir-se e andar de bicicleta ${ }^{12}$.

No entanto, ainda há uma limitação em relação à prótese mecânica no que diz respeito ao movimento de pinça. O presente estudo mostrou que não houve melhora nessa função, o que é observado na literatura, concluindo que as forças de preensão podem ser muito mais eficazes se todos os dedos forem utilizados ao mesmo tempo, podendo atingir forças de compressão razoáveis para executar atividades diárias ${ }^{19}$ da vida.

\section{CONCLUSÃO}

Pode-se concluir que a prótese confeccionada em impressora 3D se mostrou uma excelente alternativa para protetização, com boa adaptação e reposição, assim como o tratamento fisioterapêutico com a prótese mecânica permitiu ao paciente amputado aumento na sua independência e o manuseio de objetos, que não eram possíveis antes. Porém, ainda é necessária a realização de mais pesquisas sobre o tratamento fisioterapêutico em pacientes amputados que possam fazer uso da prótese mecânica feita através da impressão 3D, com o objetivo de validar protocolos terapêuticos eficazes para a melhora da funcionalidade desses pacientes.

\section{REFERÊNCIAS}

1. Montiel A, Vargas MAO, Leal SMC. Caracterização de pessoas submetidas à amputação. Enferm Foco 2012 Set;3(4):169-73.

2. Bezerra JA. Protetização de paciente amputado em nível transtibial (estudo de caso). Tese [Graduação em Fisioterapia] - Centro Universitário do Estado do Pará; 2011.

3. Resende MC, Cunha CPB, Silva AP, Sousa SJ. Rede de relações e satisfação com a vida em pessoas 
com amputação de membros. Ciências \& Cognição 2007;10:164-77.

4. Benedetto KM, Forgione MCR, Alves VLR. Reintegração corporal em pacientes amputados e a dor-fantasma. Acta fisiatrica 2002;9(2):85-89.

5. O'Sullivan SB, Schmitz TJ. Fisioterapia: avaliação e tratamento. 4. ed. São Paulo: Manole; 2004.

6. Pedrinelli A. Tratamento do paciente com amputação. São Paulo: Roca; 2010.

7. Queiroz WF. Desenvolvimento de métodos construtivos e de novos materiais empregados na confecção de cartuchos de próteses de membros inferiores. Tese [Doutorado em Engenharia mecânica] - Universidade Federal do Rio Grande do Norte 2008.

8. Both JE, Badke MR, Daandels N, Hepp DR, Santos AM. Acompanhamento de paciente com amputação de membro superior: Um Estudo de Caso. Rev Contexto Saúde 2011 Jan/Jun;10(20):611-16.

9. Ecycle [homepage na internet]. PLA: o plástico biodegradável e compostável. Disponível em: http://www.ecycle.com.br/component/content/ article/37/738-pla-o-plastico-compostavel.html.

10. Matozinhos IP, Madureira AAC, Silva GF, Madeira GCC, Oliveira IFA, Corrêa CR. Impressão 3d: inovações no campo da medicina. Revista Interdisciplinar Ciências Médicas 2017;1(1):143-62.

11. Debastiani JC. Avaliação do equilíbrio e funcionalidade em indivíduos com amputação de membro inferior protetizados e reabilitados. Tese [Graduação em Fisioterapia] - Universidade Estadual do Oeste do Paraná; 2005.

12. Guisheng Xu MD, et al. Three-dimensional-printed upper limb prosthesis for a child with traumatic amputation of right wrist a case report. Medicine 2017;95(52):1-5.

13. Maggi LE, Carolina VDA, Curado ALCF, Lemes TT. Ficha de avaliação fisioterapêutica padronizada aplicada a deficientes físicos amputados. Revista movimenta 2010;3(4):150-62.

14. Hislop HJ, Montgomery J. Provas de função muscular: técnicas de exame manual. 8. ed. Elsevier, 2008.

15. Cavado NS, Alouche SR. Instrumentos de avaliação da função de membros superiores após acidente vascular encefálico: uma revisão sistemática. Fisioter Pesq 2010;17(2):178-83.

16. Brasil. Ministério da Saúde. Diretrizes de atenção à pessoa amputada. 2013

17. Reissman T, Halsne E, Lipschutz R, Miller L, Kuiken T. A novel gel liner system with embedded electrodes for use with upper limb myoelectric prostheses. Plos one 2018 Jun;13(6):1-15.

18. Magnusson L, et al. Quality of life of prosthetic and orthotic users in South India: a cross-sectional study. Health qual life outcomes 2019;17(50):1-12.

19. Cuellar JS, Smit G, Zadpoor AA, Breedveld P. Ten guidelines for the design of non-assembly mechanisms: the case of $3 \mathrm{~d}$-printed prosthetic hands. Proc Inst Mech Eng (H) 2018;232(9):962-71.

Recebido em: 24/06/2019 Aceito em: 11/09/2019 